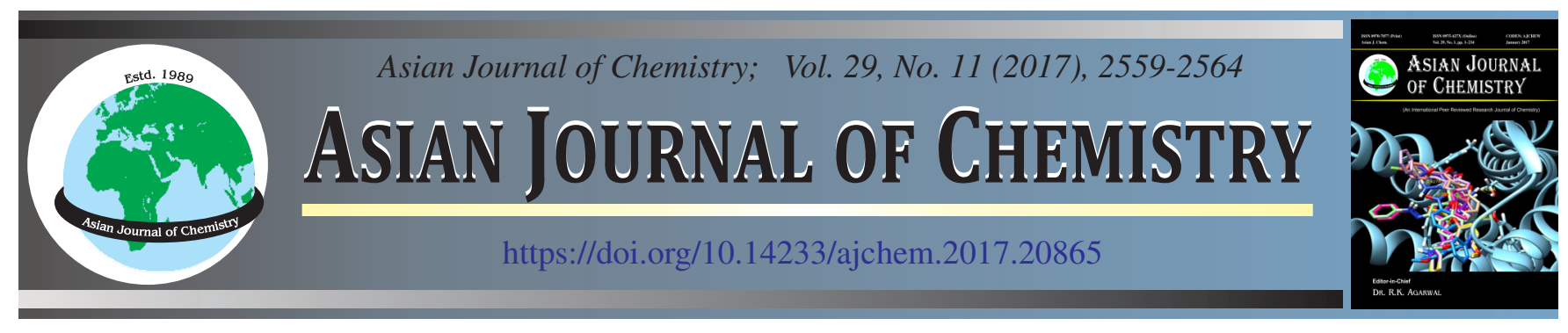

\title{
Design, Synthesis, Molecular Docking and Biological Evaluation of Novel Coumarin-Oxime Ether Derivatives as COX-2 Inhibitors
}

\author{
M. Vijaya Bhargavi ${ }^{1,2, *}$, P. Shashikala ${ }^{2}$, M. Sumakanth ${ }^{1}$ and Shravan Kumar Gunda ${ }^{2}$
}

${ }^{1}$ RBVRR Women's College of Pharmacy, 3-4-343, Barkathpura, Hyderabad-500 027, India

${ }^{2}$ Department of Pharmacy, Faculty of Technology, Osmania University, Hyderabad-500 007, India

*Corresponding author: E-mail: mvijayabhargavi@gmail.com

\begin{abstract}
Coumarin-oxime ether derivatives (14-25) were synthesized by an efficient and straight forward procedure from the reaction of 3-acetyl coumarin (1) and $o$-substituted benzyl hydroxyl amines (2-13) in pyridinium $p$-toluenesulfonate/dichloromethane (PPTS/DCM) at reflux temperature. High yields and simple operations are important features of this methodology. The method is very useful for the construction of many biologically active oxime ether derivatives. The structures of the synthesized compounds are established based on IR, NMR and MASS spectrometry. Molecular docking studies were performed against selective COX-2 enzyme using Discovery Studio v3.5. The compounds with good LibDock score were screened for their in vivo anti-inflammatory activity by paw edema method, employing indomethacin as a reference standard.
\end{abstract}

Keywords: 3-Acetyl coumarin, Benzyl hydroxylamines, Oxime ethers, Docking, COX-2.

\section{INTRODUCTION}

Coumarin and its derivatives are some of the most important oxygen heterocycles and are extensively found in various natural and synthetic products [1]. These compounds are broadly classified as, simple coumarins, furanocoumarins, pyranocoumarins, biscoumarins, triscoumarins and coumarinolignans. They are effective pharmacophores, widely used in the drug design and synthesis of novel bioactive compounds [2]. They possess different biological activities such as anticoagulant and cardiovascular activities [3], antimicrobial activities [4], anticancer [5], anti-inflammatory and antioxidant [6], antiviral [7] and enzyme-inhibition effect [8]. At this juncture, fused coumarin derivatives have attracted attention because of their biological properties [9], like antiproliferative [10], antiinflammatory [11] activities.

The oxime ether name is an abbreviation of oxy-imine ether. The oxime ether moiety (Fig. 1) is a privileged group in chemistry due to its presence in a large number of medicinal scaffolds that exhibit a broad range of biological and pharmaceutical properties, such as antifungal [12] antibacterial [13], anti-enteroviral [14], antiprotozoal [15], anti-inflammatory [16,17], anticonvulsant [18], anticancer [19], antitumor [20] activities. Therefore, oxime-ether moiety is usually chosen as an efficiently active group in the drug discovery.<smiles>[R]C=NO[R]</smiles>

$\mathbf{a}$<smiles>[R]ON=C([R])[R]</smiles>

b
Fig. 1. General structure of aldoximes (a) and ketoxime $o$-ethers (b)

However, up to date, oxime-ethers containing coumarin moiety has not been reported much. With the aim to seek new oxime-ether with high anti-inflammatory activity, here we introduced oxime-ether moiety into coumarin structure according to the sub-structure link way and synthesized twelve novel coumarin oxime-ethers. The structures of the synthesized compounds were assigned on the basis of spectral analysis and elemental analysis. The compounds were evaluated for their anti-inflammatory activity.

\section{EXPERIMENTAL}

There are two methods for the synthesis of oxime-ethers $[21,22]$. In the present study, we have synthesized a novel series of coumarin-oxime ethers (14-25) via condensation reaction of 3-acetyl coumarin (1) and $o$-substituted benzylhydroxylamines (2-13) in PPTS/DCM (pyridinium $p$-toluenesulfonate/ dichloromethane) at reflux temperature in a single step shown in Scheme-I. 


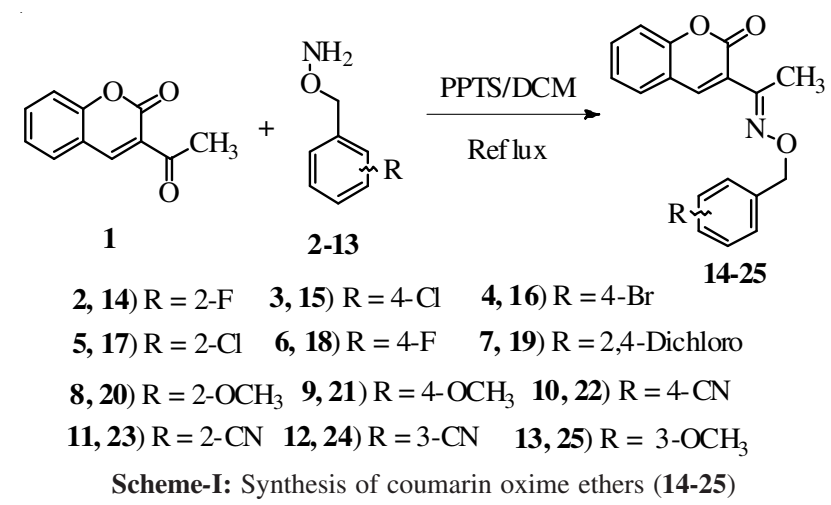

Initially, 3-acetyl coumarin (1) was synthesized from the reaction of salicyaldehyde and ethyl acetoacetate in the presence of pyridine and ethyl alcohol. N-Hydroxy phthalimide was alkylated with substituted benzyl halides in the presence of $\mathrm{K}_{2} \mathrm{CO}_{3}$ and $\mathrm{DMF}$ at room temperature. These intermediates are reacted with hydrazine hydrate in ethanol at reflux temperature to give $o$-(substituted benzyl)hydroxylamines (2-13) [23]. Coumarin oxime ethers (14-25) were synthesized from the reaction of 3-acetyl coumarin (1) and $o$-substituted benzylhydroxylamines (2-13) in PPTS/DCM at reflux temperature.

General procedure for the synthesis of compounds (1425): To a stirred solution of 3-acetyl coumarin (1) $(150 \mathrm{mg}$, $0.79 \mathrm{mmol})$ in DCM $(15 \mathrm{~mL})$ was added to PPTS $(240 \mathrm{mg}$, $0.95 \mathrm{mmol}$ ) along with O-(2-fluorobenzyl)hydroxylamine (2) $(134 \mathrm{mg}, 0.95 \mathrm{mmol})$ and refluxed continued for $3 \mathrm{~h}$. After completion of the reaction by TLC detection, Water $(30 \mathrm{~mL})$ and DCM $(15 \mathrm{~mL})$ were added and layers separated, dried over $\mathrm{Na}_{2} \mathrm{SO}_{4}$ and concentrated to yield the crude oxime ether, which was further purified by column chromatography using silca gel $(60 \times 120)$ by eluting with pet. ether:ethyl acetate $(9: 1)$ to yield pure compound.

3-(1-(2-Fluorobenzyloxyimino)ethyl)coumarin (14): Yield: $90 \%$; white solid; m.p.: $137-138^{\circ} \mathrm{C}$; $\mathrm{IR}\left(\mathrm{KBr}, v_{\max }, \mathrm{cm}^{-1}\right)$ : 2890-2910, $1721(-\mathrm{C}=\mathrm{O}), 1609(-\mathrm{C}=\mathrm{N}), 1039(\mathrm{~N}-\mathrm{O}){ }^{1}{ }^{1} \mathrm{H}$ NMR $\left(\mathrm{CDCl}_{3}, 400 \mathrm{MHz}\right) \delta 2.28\left(\mathrm{~s},-\mathrm{CH}_{3}\right), 5.30\left(\mathrm{~s},-\mathrm{OCH}_{2}\right), 7.16-$ 7.17 (m, H-5' \& H-6'), 7.28-7.30 (m, H-6, H-3' \& H-8), 7.427.46 (m, H-7), 7.52-7.54 (m, H-5 \& H-4'), 7.87 (s, H-4); ${ }^{13} \mathrm{C}$ $\operatorname{NMR}\left(\mathrm{CDCl}_{3}, 100.6 \mathrm{MHz}\right): \delta 11.5\left(-\mathrm{CH}_{3}\right), 70.2\left(-\mathrm{OCH}_{2}\right), 115.2$ (C-3'), 115.3 (C-4a), 116.1 (C-8), 118.1 (C-8a), 124.5 (C-5'), 125.2 (C-3), 125.4 (C-6), 127.9 (C-5), 128.4 (C-7), 128.7 (C-6'), $128.9\left(\mathrm{C}-1^{\prime}\right), 129.2\left(\mathrm{C}-4^{\prime}\right), 137.0(\mathrm{C}-4), 159.5\left(\mathrm{C}-2^{2}\right), 163.2(-\mathrm{C}=\mathrm{N})$, 166.0 (C-2), DIPMS: $m / z$ at $312.3(\mathrm{M}+1)$, Anal. calcd. (\%) for $\mathrm{C}_{18} \mathrm{H}_{14} \mathrm{NO}_{3} \mathrm{~F}: \mathrm{C}, 69.45 ; \mathrm{H}, 4.53 ; \mathrm{N}, 4.50$. Found: $\mathrm{C}, 69.54 ; \mathrm{H}$, $4.51 ; \mathrm{N}, 4.56$.

3-(1-(4-Chlorobenzyloxyimino)ethyl)coumarin (15): Yield: $95 \%$; white solid; m.p.: $173-174{ }^{\circ} \mathrm{C}$; IR $\left(\mathrm{KBr}, \mathrm{v}_{\max }\right.$, $\left.\mathrm{cm}^{-1}\right)$ : 2892-2912, $1723(-\mathrm{C}=\mathrm{O}), 1607(-\mathrm{C}=\mathrm{N}), 1041(\mathrm{~N}-\mathrm{O})$; ${ }^{1} \mathrm{H} \mathrm{NMR}\left(\mathrm{CDCl}_{3}, 400 \mathrm{MHz}\right): \delta 2.31\left(\mathrm{~s},-\mathrm{CH}_{3}\right), 5.24\left(\mathrm{~s},-\mathrm{OCH}_{2}\right)$, 7.28-7.41 (m, H-2' to H-6', H-6 \& H-8), 7.52-7.54 (m, H-5 \& $\mathrm{H}-7), 7.87$ (s, H-4); ${ }^{13} \mathrm{C} \mathrm{NMR}\left(\mathrm{CDCl}_{3}, 100.6 \mathrm{MHz}\right): \delta 11.5\left(-\mathrm{CH}_{3}\right)$, $77.0\left(-\mathrm{OCH}_{2}\right), 115.3$ (C-4a), 116.1 (C-8), 118.1 (C-8a), 125.4 (C-6), 125.5 (C-3), 127.9 (C-5), 128.3 (C-7), 129.0 (C-6'), 128.9 (C-3' \& C-5'), 129.7 (C-2' \& C-6'), 133.2 (C-4'), 135.4 (C-1'), $137.0(\mathrm{C}-4), 163.2(-\mathrm{C}=\mathrm{N}), 166.0$ (C-2); DIPMS: $\mathrm{m} / \mathrm{z}$ at $328.7(\mathrm{M}+1)$, Anal. calcd. (\%) for $\mathrm{C}_{18} \mathrm{H}_{14} \mathrm{NO}_{3} \mathrm{Cl}: \mathrm{C}, 65.96 ; \mathrm{H}$, 4.31; N, 4.27. Found: C, 65.95; H, 4.33; N, 4.26.
3-(1-(4-Bromobenzyloxyimino)ethyl)coumarin (16): Yield: $95 \%$; white solid; m.p.: $213-214{ }^{\circ} \mathrm{C}$; IR (KBr, $v_{\max }$, $\left.\mathrm{cm}^{-1}\right)$ : 2884-2892, $1725(-\mathrm{C}=\mathrm{O}), 1604(-\mathrm{C}=\mathrm{N}), 1042(\mathrm{~N}-\mathrm{O})$; ${ }^{1} \mathrm{H}$ NMR $\left(\mathrm{CDCl}_{3}, 400 \mathrm{MHz}\right): \delta 2.27\left(\mathrm{~s},-\mathrm{CH}_{3}\right), 5.17\left(\mathrm{~s},-\mathrm{OCH}_{2}\right)$, 7.26-7.34 (m, H-2', H-6' \& H-6, H-8), 7.48-7.56 (m, H-5, H$\left.3^{\prime} \& \mathrm{H}-7, \mathrm{H}-5^{\prime}\right), 7.83$ (s, H-4); ${ }^{13} \mathrm{C}$ NMR $\left(\mathrm{CDCl}_{3}, 100.6 \mathrm{MHz}\right)$ : $\delta 11.6\left(-\mathrm{CH}_{3}\right), 77.2\left(-\mathrm{OCH}_{2}\right), 115.1(\mathrm{C}-4 \mathrm{a}), 116.1(\mathrm{C}-8), 118.1$ (C-8a), 122.0 (C-4'), 125.4 (C-6), $125.5(\mathrm{C}-3), 127.9(\mathrm{C}-5), 128.3$ (C-7), 129.0 (C-6'), 129.3 (C-2' \& C-6'), 131.8 (C-3' \& C-5'), 136.3 (C-1'), $137.0(\mathrm{C}-4), 162.8(-\mathrm{C}=\mathrm{N}), 166.0$ (C-2); DIPMS: $\mathrm{m} / \mathrm{z}$ at $373.2(\mathrm{M}+1), 375.2(\mathrm{M}+3)$; Anal. calcd. $(\%)$ for $\mathrm{C}_{18} \mathrm{H}_{14} \mathrm{NO}_{3} \mathrm{Br}: \mathrm{C}, 58.08 ; \mathrm{H}, 3.79 ; \mathrm{N}, 3.76$. Found: C, 58.11; H, $3.83 ; \mathrm{N}, 3.79$.

3-(1-(2-Chlorobenzyloxyimino)ethyl)coumarin (17): Yield: $90 \%$; white solid; m.p.: $175-176{ }^{\circ} \mathrm{C}$; IR $\left(\mathrm{KBr}, \mathrm{v}_{\max }\right.$, $\left.\mathrm{cm}^{-1}\right)$ : 2892-2912, $1720(-\mathrm{C}=\mathrm{O}), 1610(-\mathrm{C}=\mathrm{N}), 1038(\mathrm{~N}-\mathrm{O})$; ${ }^{1} \mathrm{H} \mathrm{NMR}\left(\mathrm{CDCl}_{3}, 400 \mathrm{MHz}\right): \delta 2.28\left(\mathrm{~s},-\mathrm{CH}_{3}\right), 5.30\left(\mathrm{~s},-\mathrm{OCH}_{2}\right)$, 7.16-7.17 (m, H-5' \& H-6'), 7.28-7.30 (m, H-6, H-3' \& H-8), 7.42-7.46 (m, H-7), 7.52-7.54 (m, H-5 \& H-4'), 7.87 (s, H-4); ${ }^{13} \mathrm{C}$ NMR $\left(\mathrm{CDCl}_{3}, 100.6 \mathrm{MHz}\right): \delta 11.5\left(-\mathrm{CH}_{3}\right), 71.9\left(-\mathrm{OCH}_{2}\right)$, 115.3 (C-4a), 116.1 (C-8), 118.1 (C-8a), 125.2 (C-3), 125.4 (C-6), 127.0 (C-5'), 127.9 (C-5), 128.3 (C-7), 128.5 (C-6'), 129.0 (C-3'), 128.9 (C-4'), $130.7\left(\mathrm{C}-2^{\prime}\right), 137.0(\mathrm{C}-4), 138.2\left(\mathrm{C}-1^{\prime}\right), 163.2(-\mathrm{C}=\mathrm{N})$, 166.0 (C-2), DIPMS: $m / z$ at 328.7 (M+1), Anal. calcd. (\%) for $\mathrm{C}_{18} \mathrm{H}_{14} \mathrm{NO}_{3} \mathrm{ClF}: \mathrm{C}, 65.96 ; \mathrm{H}, 4.31 ; \mathrm{N}, 4.27$. Found: C, 65.54; $\mathrm{H}, 4.41 ; \mathrm{N}, 4.26$.

3-(1-(4-Fluorobenzyloxyimino)ethyl)coumarin (18): Yield: $95 \%$; white solid; m.p.: $191-192{ }^{\circ} \mathrm{C}$; IR ( $\mathrm{KBr}, v_{\max }$, $\left.\mathrm{cm}^{-1}\right)$ : 2896-2918, $1725(-\mathrm{C}=\mathrm{O}), 1609(-\mathrm{C}=\mathrm{N}), 1045(\mathrm{~N}-\mathrm{O})$; ${ }^{1} \mathrm{H} \mathrm{NMR}\left(\mathrm{CDCl}_{3}, 400 \mathrm{MHz}\right): \delta 2.26\left(\mathrm{~s},-\mathrm{CH}_{3}\right), 5.18\left(\mathrm{~s},-\mathrm{OCH}_{2}\right)$, 7.04-7.08 (m, H-5' \& H-6'), 7.33-7.39 (m, H-6, H-3' \& H-8), 7.52-7.54 (H-7 \& H-2'), 7.64-7.69 (m, H-5), 7.86 (s, H-4) ${ }^{13} \mathrm{C}$ $\operatorname{NMR}\left(\mathrm{CDCl}_{3}, 100.6 \mathrm{MHz}\right): \delta 11.5\left(-\mathrm{CH}_{3}\right), 77.0\left(-\mathrm{OCH}_{2}\right), 115.3$ (C-4a), 115.7 (C-3' \& C-5'), 116.1 (C-8), 118.1 (C-8a), 125.4 (C-6), 125.2 (C-3), 127.9 (C-5), 128.3 (C-7), 128.7 (C-6'), 128.7 (C-2' \& C-6'), $132.9\left(\mathrm{C}-1^{\prime}\right), 137.0(\mathrm{C}-4), 161.8\left(\mathrm{C}-4^{\prime}\right), 163.2(-\mathrm{C}=\mathrm{N})$, 166.1 (C-2); DIPMS: $m / z$ at $328.7(\mathrm{M}+1)$, Anal. calcd. (\%) for $\mathrm{C}_{18} \mathrm{H}_{14} \mathrm{NO}_{3} \mathrm{~F}$ : C, $69.45 ; \mathrm{H}, 4.53 ; \mathrm{N}, 4.50$. Found: $\mathrm{C}, 69.55$; $\mathrm{H}, 4.43 ; \mathrm{N}, 4.46$.

3-(1-(2,4-Dichlorobenzyloxyimino)ethyl)coumarin (19): Yield: $90 \%$; white solid; m.p.: $215-216^{\circ} \mathrm{C}$; IR ( $\mathrm{KBr}$, $\left.v_{\max }, \mathrm{cm}^{-1}\right): 2890-2910,1729(-\mathrm{C}=\mathrm{O}), 1611(-\mathrm{C}=\mathrm{N}), 1031(\mathrm{~N}-\mathrm{O})$; ${ }^{1} \mathrm{H} \mathrm{NMR}\left(\mathrm{CDCl}_{3}, 400 \mathrm{MHz}\right): \delta 2.29\left(\mathrm{~s},-\mathrm{CH}_{3}\right), 5.31\left(\mathrm{~s},-\mathrm{OCH}_{2}\right)$, 7.26-7.29 (m, H-8, H-5' \& H-6'), 7.35-7.41 (m, H-3' \& H-7), 7.52-7.57 (m, H-5 \& H-6), 7.86 (s, H-4); ${ }^{13} \mathrm{C}$ NMR $\left(\mathrm{CDCl}_{3}\right.$, $100.6 \mathrm{MHz}): \delta 11.5\left(-\mathrm{CH}_{3}\right), 71.9\left(-\mathrm{OCH}_{2}\right), 115.3(\mathrm{C}-4 \mathrm{a}), 116.1$ (C-8), 118.1 (C-8a), 125.2 (C-3), 125.4 (C-6), 127.1 (C-5'), 127.9 (C-5), 128.3 (C-7), 129.9 (C-6'), 130.6 (C-3'), 133.8 (C-2'), $134.6\left(\mathrm{C}-4^{\prime}\right), 136.3\left(\mathrm{C}-1^{\prime}\right), 137.0(\mathrm{C}-4), 163.2(-\mathrm{C}=\mathrm{N})$, 166.0 (C-2), DIPMS: $m / z$ at $363.2(\mathrm{M}+1)$, Anal. calcd. (\%) for $\mathrm{C}_{18} \mathrm{H}_{13} \mathrm{NO}_{3} \mathrm{Cl}_{2}: \mathrm{C}, 59.69 ; \mathrm{H}, 3.62 ; \mathrm{N}, 3.87$. Found: C, 59.54; $\mathrm{H}, 3.61 ; \mathrm{N}, 3.96$.

3-(1-(2-Methoxybenzyloxyimino)ethyl)coumarin (20): Yield: $85 \%$; white solid; m.p.: $142-144{ }^{\circ} \mathrm{C}$; IR $\left(\mathrm{KBr}, v_{\max }\right.$, $\left.\mathrm{cm}^{-1}\right)$ : 2892-2912, $1723(-\mathrm{C}=\mathrm{O}), 1610(-\mathrm{C}=\mathrm{N}), 1041(\mathrm{~N}-\mathrm{O})$; ${ }^{1} \mathrm{H} \mathrm{NMR}\left(\mathrm{CDCl}_{3}, 400 \mathrm{MHz}\right): \delta 2.38\left(\mathrm{~s},-\mathrm{CH}_{3}\right), 3.83\left(\mathrm{~s},-\mathrm{OCH}_{3}\right)$, $5.30\left(\mathrm{~s},-\mathrm{OCH}_{2}\right), 6.92-6.96$ (m, H-3', H-4' \& H-5'), 7.25 (m, H-6'), 7.40-7.44 (m, H-6), 7.51 (dd, $J=1.09 \mathrm{~Hz}, 8.05 \mathrm{~Hz}, \mathrm{H}-8)$, 7.65 (m, H-7), 7.54 (s, H-4), 7.84 (dd, $J=1.61 \mathrm{~Hz}, 7.72 \mathrm{~Hz}$, 
$\mathrm{H}-5) ;{ }^{13} \mathrm{CNMR}\left(\mathrm{CDCl}_{3}, 100.6 \mathrm{MHz}\right): \delta 11.5\left(-\mathrm{CH}_{3}\right), 71.1\left(-\mathrm{OCH}_{2}\right)$, 115.3 (C-4a), 116.1 (C-8), 118.1 (C-8a), $124.0(\mathrm{C}-5$ '), 125.2 (C-3), 125.4 (C-6), 127.2 (C-1'), 127.9 (C-5), 128.3 (C-7), 128.6 (C-4'), 129.0 (C-3'), 129.7 (C-6'), 137.0 (C-4), 156.7 $\left(\mathrm{C}-2{ }^{\prime}\right), 163.2(-\mathrm{C}=\mathrm{N}), 166.0(\mathrm{C}-2)$; DIPMS: $\mathrm{m} / 2$ at $324.3(\mathrm{M}+1)$, Anal. calcd. (\%) for $\mathrm{C}_{19} \mathrm{H}_{17} \mathrm{NO}_{4}: \mathrm{C}, 70.58 ; \mathrm{H}, 5.30 ; \mathrm{N}, 4.33$. Found: C, 70.54; H, 5.41; N, 4.36.

3-(1-(4-Methoxybenzyloxyimino)ethyl)coumarin (21): Yield: $95 \%$; white solid; m.p.: 213-214 ${ }^{\circ} \mathrm{C}$; IR (KBr, $\mathrm{v}_{\max }$, $\left.\mathrm{cm}^{-1}\right)$ : 2888-2908, $1721(-\mathrm{C}=\mathrm{O}), 1609(-\mathrm{C}=\mathrm{N}), 1042(\mathrm{~N}-\mathrm{O})$; ${ }^{1} \mathrm{H} \mathrm{NMR}\left(\mathrm{CDCl}_{3}, 400 \mathrm{MHz}\right): \delta 2.34\left(\mathrm{~s},-\mathrm{CH}_{3}\right), 5.21\left(\mathrm{~s},-\mathrm{OCH}_{2}\right)$, 6.96-7.01 (m, H-2', H-3' \& H-5', H-6'), 7.42-7.46 (m, H-6 \& H-8), 7.54 (s, H-4), 7.65-7.69 (m, H-7), 7.84 (dd, $J=1.61 \mathrm{~Hz}$, $7.72 \mathrm{~Hz}, \mathrm{H}-5) ;{ }^{13} \mathrm{C} \mathrm{NMR}\left(\mathrm{CDCl}_{3}, 100.6 \mathrm{MHz}\right): \delta 11.5\left(-\mathrm{CH}_{3}\right)$, $77.0\left(-\mathrm{OCH}_{2}\right), 114.5$ (C-3' \& C-5'), 115.3 (C-4a), 116.1 (C-8), 118.1 (C-8a), 125.2 (C-3), 125.4 (C-6), 127.9 (C-5), 128.3 (C-7), 129.3 (C-2' \& C-6'), 129.6 (C-1'), 137.0 (C-4), 159.5 (C-4'), $163.2(-\mathrm{C}=\mathrm{N}), 166.0(\mathrm{C}-2)$; DIPMS: $m / z$ at $324.3(\mathrm{M}+1)$, Anal. calcd. $(\%)$ for $\mathrm{C}_{19} \mathrm{H}_{17} \mathrm{NO}_{4}$ : C, 70.58; H, 5.31; N, 4.33. Found: C, $70.65 ; \mathrm{H}, 5.33 ; \mathrm{N}, 4.26$.

3-(1-(4-Cyanobenzyloxyimino)ethyl)coumarin (22): Yield: $85 \%$; white solid; m.p.: 223-224 ${ }^{\circ} \mathrm{C}$; IR (KBr, $v_{\max }$, $\left.\mathrm{cm}^{-1}\right)$ : 2892-2912, $1723(-\mathrm{C}=\mathrm{O}), 1607(-\mathrm{C}=\mathrm{N}), 1041(\mathrm{~N}-\mathrm{O})$, $2230(-\mathrm{CN}) ;{ }^{1} \mathrm{H}$ NMR $\left(\mathrm{CDCl}_{3}, 400 \mathrm{MHz}\right): \delta 2.37\left(\mathrm{~s},-\mathrm{CH}_{3}\right)$, $5.22\left(\mathrm{~s},-\mathrm{OCH}_{2}\right), 7.32-7.39$ (m, H-2', H-6', \& H-6), 7.44 (dd, $J=1.09 \mathrm{~Hz}, J=8.05 \mathrm{~Hz}, \mathrm{H}-5$ \& H-7), 7.65-7.71 (m, H-7), $7.82\left(\mathrm{dd}, J=2.23 \mathrm{~Hz}, J=8.29 \mathrm{~Hz}, \mathrm{H}-3^{\prime} \& \mathrm{H}-5 '\right), 7.87$ (s, H-4), $8.03(\mathrm{dd}, J=1.61 \mathrm{~Hz}, 7.72 \mathrm{~Hz}, \mathrm{H}-5) ;{ }^{13} \mathrm{C} \mathrm{NMR}\left(\mathrm{CDCl}_{3}, 100.6\right.$ $\mathrm{MHz}): \delta 11.5\left(-\mathrm{CH}_{3}\right), 77.0\left(-\mathrm{OCH}_{2}\right), 111.5\left(\mathrm{C}-4{ }^{\prime}\right), 115.3(\mathrm{C}-4 \mathrm{a})$, 116.1 (C-8), 118.1 (C-8a), 118.6 (4'-CN), 125.4 (C-6), 125.5 (C-3), 127.9 (C-5), 128.3 (C-7), 127.9 (C-2' \& C-6'), 132.4 (C-3' \& C-5'), $141.6\left(\mathrm{C}-1^{\prime}\right), 137.0(\mathrm{C}-4), 163.2(-\mathrm{C}=\mathrm{N}), 166.0(\mathrm{C}-2)$; DIPMS: $m / z$ at $319.3(\mathrm{M}+1)$, Anal. calcd. $(\%)$ for $\mathrm{C}_{19} \mathrm{H}_{14} \mathrm{~N}_{2} \mathrm{O}_{3}$ : C, 71.69; H, 4.43; N, 8.80. Found: C, 71.95; H, 4.41; N, 8.82.

3-(1-(2-Cyanobenzyloxyimino)ethyl)coumarin (23): Yield: $80 \%$; white solid; m.p.: $153-155^{\circ} \mathrm{C}$; IR (KBr, $v_{\max }$, $\left.\mathrm{cm}^{-1}\right)$ : 2895-2915, $1727(-\mathrm{C}=\mathrm{O}), 1607(-\mathrm{C}=\mathrm{N}), 1041(\mathrm{~N}-\mathrm{O})$, $2233(-\mathrm{CN}) ;{ }^{1} \mathrm{H}$ NMR $\left(\mathrm{CDCl}_{3}, 400 \mathrm{MHz}\right): \delta 2.30\left(\mathrm{~s},-\mathrm{CH}_{3}\right)$, $5.31\left(\mathrm{~s},-\mathrm{OCH}_{2}\right), 7.16-7.17$ (m, H-5' \& H-6'), 7.28-7.31 (m, H-6, H-3' \& H-8), 7.42-7.46 (m, H-7), 7.51-7.56 (m, H-5 \& H-4'), 7.89 (s, H-4); ${ }^{13} \mathrm{C} \mathrm{NMR}\left(\mathrm{CDCl}_{3}, 100.6 \mathrm{MHz}\right): \delta 11.9\left(-\mathrm{CH}_{3}\right)$, $70.6\left(-\mathrm{OCH}_{2}\right), 115.3(\mathrm{C}-3$ '), 115.7 (C-4a), 116.1 (C-8), $119.8(\mathrm{C}-8 \mathrm{a})$, 124.7 (C-5'), 125.3 (C-3), 125.8 (C-6), 127.9 (C-5), 128.3 (C-7), 128.7 (C-6'), 128.9 (C-1'), 129.6 (C-4'), 137.3 (C-4), 159.6 (C-2'), $163.4(-\mathrm{C}=\mathrm{N}), 166.0(\mathrm{C}-2)$, DIPMS: $\mathrm{m} / \mathrm{z}$ at $319.3(\mathrm{M}+1)$, Anal. calcd. (\%) for $\mathrm{C}_{19} \mathrm{H}_{14} \mathrm{~N}_{2} \mathrm{O}_{3}$ : C, 71.69; H, 4.43; N, 8.80. Found: C, 71.54; H, 4.49; N, 8.76.

3-(1-(3-Cyanobenzyloxyimino)ethyl)coumarin (24): Yield: $75 \%$; white solid; m.p.: $192-193{ }^{\circ} \mathrm{C}$; IR (KBr, $v_{\max }$, $\left.\mathrm{cm}^{-1}\right)$ : 2891-2911, 1729 (-C=O), $1608(-\mathrm{C}=\mathrm{N}), 1049(\mathrm{~N}-\mathrm{O})$, $2236(-\mathrm{CN}) ;{ }^{1} \mathrm{H}$ NMR $\left(\mathrm{CDCl}_{3}, 400 \mathrm{MHz}\right): \delta 2.31\left(\mathrm{~s},-\mathrm{CH}_{3}\right)$, $5.33\left(\mathrm{~s},-\mathrm{OCH}_{2}\right), 7.33-7.42$ (m, H-6 \& H-8), 7.50-7.55 (m, H-5' \& H-6'), 7.68 (td, H-7, $J=8.05 \mathrm{~Hz}, J=1.61 \mathrm{~Hz}, J=7.52 \mathrm{~Hz}$ ), 7.97 (m, H-4'), 8.01-8.03 (m, H-4 \& H-5), 8.07 (d, H-2', J = 1.55 $\mathrm{Hz}, J=1.55 \mathrm{~Hz}) ;{ }^{13} \mathrm{C} \mathrm{NMR}\left(\mathrm{CDCl}_{3}, 100.6 \mathrm{MHz}\right): \delta 11.5\left(-\mathrm{CH}_{3}\right)$, $76.5\left(-\mathrm{OCH}_{2}\right), 112.8$ (C-3'), 116.1 (C-8), 115.7 (C-4a), 118.6 (3-CN), 119.8 (C-8a), 125.3 (C-3), 125.4 (C-6), 127.7 (C-5), 128.3 (C-7), 129.7 (C-5'), 131.1 (C-4'), 131.3 (C-2'), 131.4 (C-6'),
137.0 (C-4), 141.9 (C-1'), $163.4(-\mathrm{C}=\mathrm{N}), 166.0$ (C-2); DIPMS: $\mathrm{m} / \mathrm{z}$ at $319.3(\mathrm{M}+1)$, Anal. calcd. (\%) for $\mathrm{C}_{19} \mathrm{H}_{14} \mathrm{~N}_{2} \mathrm{O}_{3}: \mathrm{C}, 71.69$; H, 4.43; N, 8.80. Found: C, 71.53; H, 4.51; N, 8.74.

3-(1-(3-Methoxybenzyloxyimino)ethyl)coumarin (25): Yield: $85 \%$; white solid; m.p.: 211-212 ${ }^{\circ} \mathrm{C}$; IR (KBr, $\mathrm{v}_{\max }$, $\left.\mathrm{cm}^{-1}\right)$ : 2895-2915, $1728(-\mathrm{C}=\mathrm{O}), 1610(-\mathrm{C}=\mathrm{N}), 1041(\mathrm{~N}-\mathrm{O})$; ${ }^{1} \mathrm{H} \mathrm{NMR}\left(\mathrm{CDCl}_{3}, 400 \mathrm{MHz}\right): \delta 2.32\left(\mathrm{~s},-\mathrm{CH}_{3}\right), 3.83\left(\mathrm{~s},-\mathrm{OCH}_{3}\right)$, $5.31\left(\mathrm{~s},-\mathrm{OCH}_{2}\right), 6.87-6.97$ (m, H-2' \& H-4'), 7.05-7.23 (m, H5' \& H-6'), 7.33-7.42 (m, H-6 \& H-8), 7.68 (td, H-7, J = 8.05 $\mathrm{Hz}, J=1.61 \mathrm{~Hz}, J=7.52 \mathrm{~Hz}), 8.01-8.03(\mathrm{~m}, \mathrm{H}-4 \& \mathrm{H}-5) ;{ }^{13} \mathrm{C}$ $\mathrm{NMR}\left(\mathrm{CDCl}_{3}, 100.6 \mathrm{MHz}\right): \delta 11.6\left(-\mathrm{CH}_{3}\right), 55.8\left(-\mathrm{OCH}_{3}\right), 77.3$ $\left(-\mathrm{OCH}_{2}\right), 113.2\left(\mathrm{C}-4{ }^{\prime}\right), 113.5(\mathrm{C}-2 '), 115.7$ (C-4a), 116.1 (C-8), 119.4 (C-6'), 119.8 (C-8a), 125.3 (C-3), 125.4 (C-6), 127.9 (C-5), 128.3 (C-7), 129.9 (C-5'), 137.0 (C-4), 142.2 (C-1'), 160.8 (C-3'), $163.2(-\mathrm{C}=\mathrm{N}), 166.5(\mathrm{C}-2)$; DIPMS: $m / z$ at $324.3(\mathrm{M}+1)$, Anal. calcd. (\%) for $\mathrm{C}_{19} \mathrm{H}_{17} \mathrm{NO}_{4}$ : C, 70.58; H, 5.30; N, 4.33. Found: C, 70.53; H, 45.31; N, 4.43.

Molecular docking studies: Computational studies of the novel coumarin oxime ether derivatives were done using Discovery studio v 3.5. The three dimensional structure of celecoxib bound at the COX-2 active site (PDB ID: 3LN1) was retrieved from the Brookhaven Protein Data Bank (PDB), USA (http://www.rcsb.org/pdb). Protein preparation and ligand preparation wizard were used for protein and ligand preparation respectively. Initially, ions, water molecules, all the internal ligands were removed and missing atoms were inserted before minimization of the target protein. Alternate conformations (disorder) were removed.

The bioactive binding poses of inhibitors in the active site of the enzyme was generated by using a LibDock program of Discovery Studio. The ligand poses were placed in to polar and apolar interactions site. MMFF force field was used for energy minimization of the ligands. 'Hotspots' are the binding site features. The hotspot map counts for polar and apolar cluster in the active site of the protein which is further used for the alignment of the ligand conformations to the interaction sites of the protein. All the minimized ligand poses and their rankings are based on the ligands score. In accordance with the LibDock score, each pose is assessed for binding energy, by a simple pairwise method. The ligands with high LibDock scores are considered for estimating binding energies of the protein-ligand complex. The complex pose with the best binding energy is used for further binding mode analysis. For the docking validation, the co-crystallized ligand CEL in the Musmusculus COX-2 binding site is redocked. The binding affinities of the synthesized compounds were compared and analyzed in reference to celecoxib to identify structural characteristics of the complexes formed by these compounds and the protein.

Anti-inflammatory activity-carrageenan-induced rat paw edma assay: Anti-inflammatory activity of synthesized compounds was assessed by carrageenan-induced rat paw edema method which was described by Winter et al. [24]. Carrageenan $(0.1 \mathrm{~mL}, 1 \%)$ was administered into the plantar surface of the right hind paw of the animals to induce edema. Wistar rats weighing between 190 and $260 \mathrm{~g}$, were used in the current study.

Indomethacin was used as a reference drug. The rats were divided into eight groups, each group consist of five animals. 
Group-1 referred as control (animals received carrageenan along with the vehicle [0.5\% carboxy methyl cellulose (CMC)] and Group- 2 received standard reference (indomethacin) along with the vehicle prior to the administration of carrageenan. Group 3-8 received the test compounds. All the test compounds were suspended in $0.5 \%$ of CMC and administered orally (10 $\mathrm{mg} / \mathrm{kg}$ ) $60 \mathrm{~min}$ prior to the injection of $0.1 \mathrm{~mL}$ of freshly prepared carrageenan $(1 \%)$ in physiological solution $(154 \mathrm{mM}$ $\mathrm{NaCl}$ ) into the sub-planter tissue of hind paw of each rat. Plethysmometer apparatus was used to measure the volume prior to the administration of carrageenan, 2 and $4 \mathrm{~h}$ after the injection. The increase in volume of the rat paw was adopted as a measure of edema. The antiedematous effects of the compounds were estimated as percentage inhibition of the induced inflammation in comparision with control. Statistical analysis was also carried out using a one-way analysis of variance (ANOVA). A significance level of $p<0.001$ denoted significance in all cases.

The percentage inhibition of edema was calculated using the formula given below:

$$
\left(\mathrm{V}_{\mathrm{c}}-\mathrm{V}_{\mathrm{t}} / \mathrm{V}_{\mathrm{c}}\right) \times 100
$$

$\mathrm{V}_{\mathrm{c}}$ is the increase in paw volume of control; $\mathrm{V}_{\mathrm{t}}$ is the increase in paw volume after administration of the test compound.

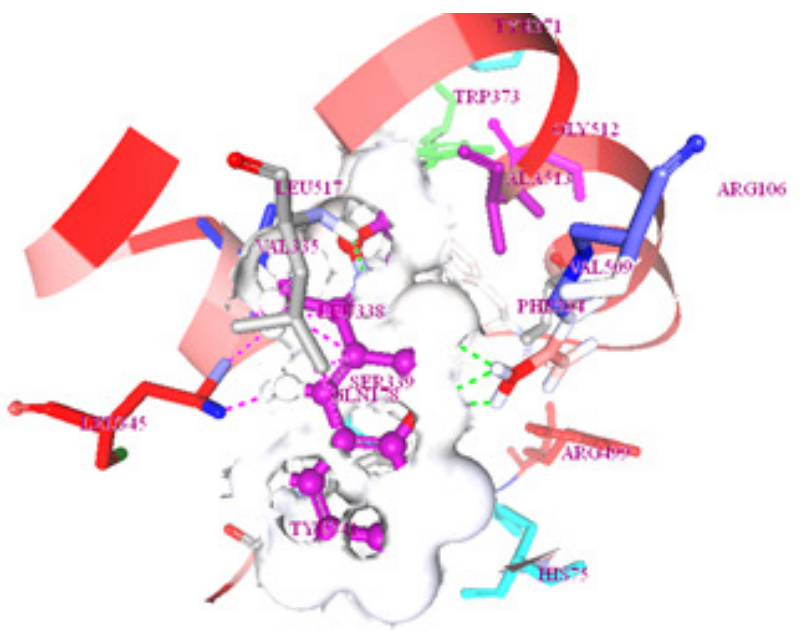

(a)

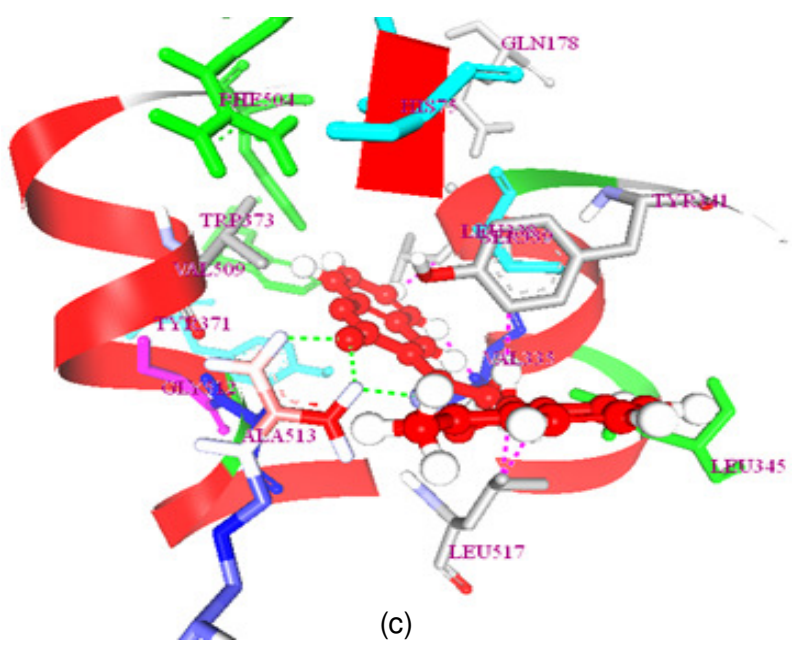

\section{RESULTS AND DISCUSSION}

Docking: Molecular docking studies are used to study the binding modes and affinities of our synthesized compounds with Musmusculus COX-2 (PDB ID: 3LN1). All the ligands were targeted to celecoxib bound at the COX-2 site. The best ligand conformation is chosen on the base of LibDock score and highly interacting amino acid residues. Of the ten conformations generated for each compound, the compound with the highest LibDock score is taken for interaction analysis of the hydrogen bonding. LibDock scores of all the compounds along with their hydrogen bond interactions and bond lengths are depicted in Table-1. The interaction of amino acid residues with bound ligands as represented by different colours e.g., pink indicates electrostatic interaction, purple indicates covalent bond and green colour indicates vander waals molecular interaction (Fig. 2). From the overall docking and interaction analysis, the best conformation of the compound 23 docked complex shows high LibDock score of $141.261 \mathrm{kcal} / \mathrm{mol}$ and forms hydrogen bonds with the protein COX-2. Hydrogen bonds are formed between the $\mathrm{H} 12$ of arginine 106 with the N24 atom of compound 23 with a hydrogen bond distance of 2.303000 $\AA$ and the second hydrogen bond is formed with the same amino acid arginine 106 between H22 with the N24 atom of compound 23 with a hydrogen bond distance of 1.976000 .

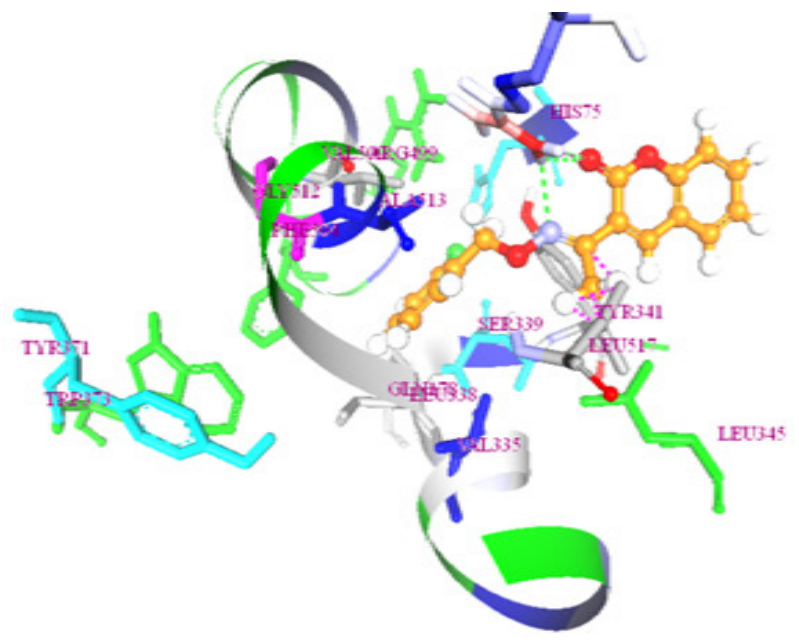

(b)

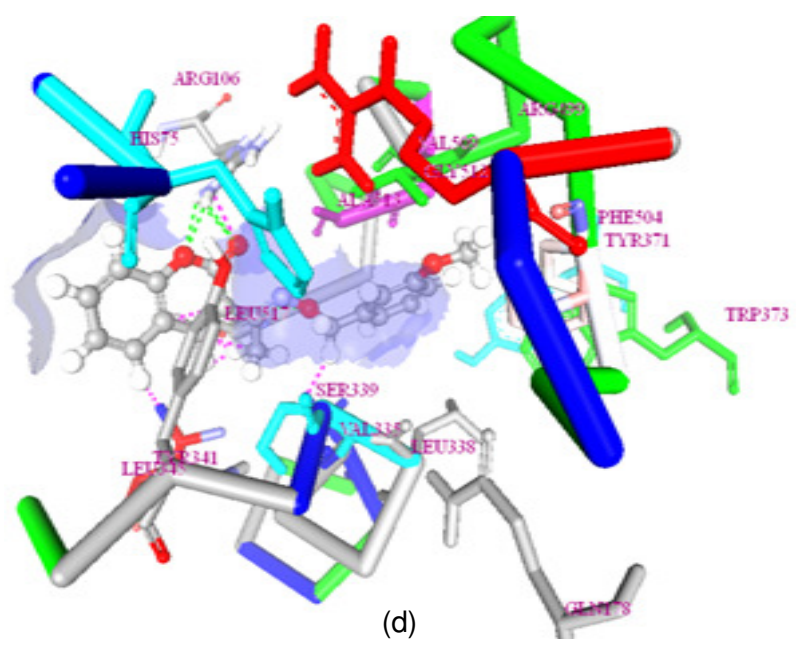

(d)

Fig. 2. Molecular interaction of 3LN1 with (a) compound 14, (b) compound 17, (c) compound 20 and (d) compound 23 


\begin{tabular}{|c|c|c|c|}
\hline \multicolumn{4}{|c|}{$\begin{array}{c}\text { TABLE-1 } \\
\text { INTERACTION OF LIGAND AND AMINO ACIDS }\end{array}$} \\
\hline Compound No. & Interacting amino acids & Interacting atoms & H-Distance \\
\hline \multirow{6}{*}{14} & \multirow{6}{*}{$\begin{array}{l}\text { Tyr371, Trp373, Phe504, Gly512 } \\
\text { Gln178, Arg499, Arg106, Leu517 } \\
\text { Leu338, Phe504His75, Val509 } \\
\text { Val335, Ala513, Leu345, Leu517 }\end{array}$} & A:ARG106:HH11- Comp14:O9 & 2.490000 \\
\hline & & A:ARG106:HH11 -Comp14:O7 & 2.467000 \\
\hline & & A:ARG106:HH12 -Comp14:O7 & 2.256000 \\
\hline & & A:LEU517:HN - Comp14:N13 & 1.836000 \\
\hline & & Comp14:H28 - A:LEU345:CD2 & 1.996000 \\
\hline & & Comp14:H30 - A:LEU345:CD1 & 2.133000 \\
\hline \multirow{6}{*}{15} & & A:ARG106:HH11 - Comp15:O9 & 2.299000 \\
\hline & Tyr371, Trp373, Phe504, Gly512 & A:ARG106:HH11 - Comp15:N13 & 2.315000 \\
\hline & Gln178, Arg499, Arg106, Leu517 & A:ARG106:HH12 - Comp15:O9 & 1.951000 \\
\hline & Leu338, Phe504His75, Val509 & A:ARG106:HH12 - Comp15:N13 & 2.099000 \\
\hline & Val335, Ala513, Leu345, Leu517 & Comp15:H30 - A:TYR341:CE2 & 2.182000 \\
\hline & & Comp15:H36 - A:VAL509:CG1 & 2.028000 \\
\hline \multirow{4}{*}{16} & Tyr371, Trp373, Phe504, Gly512 & A:ARG106:HH12 - Comp16:N13 & 2.289000 \\
\hline & Gln178, Arg499, Arg106, Leu517 & A:ARG106:HH12 - Comp16:O9 & 1.722000 \\
\hline & Leu338, Phe504His75, Val509 & A:ARG106:HH11 - Comp16:O9 & 2.214000 \\
\hline & Val335, Ala513, Leu345, Leu517 & A:ARG106:NH1 - Comp16:O9 & 2.211000 \\
\hline \multirow{5}{*}{17} & \multirow{5}{*}{$\begin{array}{l}\text { Tyr371, Trp373, Phe504, Gly512 } \\
\text { Gln178, Arg499, Arg106, Leu517 } \\
\text { Leu338, Phe504His75, Val509 } \\
\text { Val335, Ala513, Leu345, Leu517 }\end{array}$} & A:ARG106:HH12 - Comp17:N13 & 2.458000 \\
\hline & & A:ARG106:HH11 - Comp17:O9 & 2.195000 \\
\hline & & A:ARG106:HH12 - Comp17:O9 & 1.637000 \\
\hline & & Comp17:C12 - A:LEU517:CD1 & 2.572000 \\
\hline & & Comp17:C14 - A:LEU517:CG & 2.388000 \\
\hline \multirow{5}{*}{18} & \multirow{5}{*}{$\begin{array}{l}\text { Tyr371, Trp373, Phe504, Gly512 } \\
\text { Gln178, Arg499, Arg106, Leu517 } \\
\text { Leu338, Phe504His75, Val509 } \\
\text { Val335, Ala513, Leu345, Leu517 }\end{array}$} & A:ARG106:HH11 - Comp18:O7 & 2.418000 \\
\hline & & A:ARG106:HH12 - Comp18:O7 & 2.030000 \\
\hline & & A:ARG106:HH12 - Comp18:O9 & 2.457000 \\
\hline & & Comp18:H25 - A:LEU345:CD2 & 1.942000 \\
\hline & & Comp18:H35 - A:LEU338:CD2 & 1.935000 \\
\hline \multirow{4}{*}{19} & Tyr371, Trp373, Phe504, Gly512 & & \\
\hline & Gln178, Arg499, Arg106, Leu517 & A:TYR371:HH - Sc1_Comp19:Cl23 & 2.342000 \\
\hline & Leu338, Phe504His75, Val509 & Comp19:H25 - A:TYR341:CE2 & 2.158000 \\
\hline & Val335, Ala513, Leu345, Leu517 & & \\
\hline \multirow{5}{*}{20} & & A:ARG106:HH22 - Sc1_Comp20:O9 & 2.393000 \\
\hline & 1yr3/1, Irp3/3, Phesu4, Glys12 & A:ARG106:HH12 - Sc1_Comp20:O9 & 2.391000 \\
\hline & Leu338. Phe504His75. Val509 & A:ARG106:HH12 - Sc1_Comp20:N13 & 2.322000 \\
\hline & Va1335 Ala513 Jey 345 & A:TYR341:CE2 - Comp20:H34 & 1.959000 \\
\hline & & A:LEU338:CB - Comp20:H28 & 2.186000 \\
\hline \multirow{3}{*}{21} & Tyr371, Trp373, Phe504, Gly512 & A:ARG106:HH12 - Comp21:N13 & 2.378000 \\
\hline & Gln178, Arg499, Arg106, Leu517 & A:ARG106:HH11 - Comp21:N13 & 2.018000 \\
\hline & $\begin{array}{l}\text { Leu338, Phe504His75, Val509 } \\
\text { Val335, Ala513, Leu345, Leu517 }\end{array}$ & A:TYR341:CE2 - Comp21:H31 & 1.999000 \\
\hline \multirow{4}{*}{22} & Tyr371, Trp373, Phe504, Gly512 & A:TYR371:HH - Comp22:N24 & 2.344000 \\
\hline & Gln178, Arg499, Arg106, Leu517 & A:ARG106:HH11 - Comp22:O9 & 2.003000 \\
\hline & Leu338, Phe504His75, Val509 & A:ARG106:HH12 - Comp22:O9 & 1.508000 \\
\hline & Val335, Ala513, Leu345, Leu517 & Comp22:H30 - A:LEU345:CD2 & 2.141000 \\
\hline \multirow{4}{*}{23} & Tyr371, Trp373, Phe504, Gly512 & A:TYR341:HH - Comp23:N24 & 2.366000 \\
\hline & Gln178, Arg499, Arg106, Leu517 & A:ARG106:HH22 - Comp23:N24 & 1.976000 \\
\hline & Leu338, Phe504His75, Val509 & A:ARG106:HH12 - Comp23:N24 & 2.303000 \\
\hline & Val335, Ala513, Leu345, Leu517 & Comp23:C23 - A:ARG106:HH12 & 1.977000 \\
\hline \multirow{5}{*}{24} & & A:ARG106:HH12 - Comp24:N13 & 2.158000 \\
\hline & Tyr371, Trp373, Phe504, Gly512 & A:ARG106:HH12 - Comp24:O15 & 2.341000 \\
\hline & Gln178, Arg499, Arg106, Leu517 & A:ARG499:HH22 - Comp24:N24 & 2.018000 \\
\hline & Leu338, Phe504His75, Val509 & A:LEU517:HN - Comp24:O9 & 1.875000 \\
\hline & Val335, Ala513, Leu345, Leu517 & Comp24:H38 - A:TYR341:OH & 1.733000 \\
\hline & & A:LEU517:HN - Comp25:O15 & 2.279000 \\
\hline & 1yr3/1, Irp3/3, Phes04, Glys 12 & A:LEU517:HN - Comp25:N13 & 2.270000 \\
\hline 25 & Gln178, Arg499, Arg106, Leu517 & A:ARG106:HH12 - Comp25:O7 & 2.148000 \\
\hline & Leu338, Phe504His75, Val509 & A:LEU345:CD2 - Comp25:H26 & 1.984000 \\
\hline & & A:LEU517:CD1 - Comp25:C11 & 1.977000 \\
\hline & Leu345, Leu157, Arg106, & Celecoxib:H28 - A:HIS75:NE2 & 2.387000 \\
\hline & Val335, Tyr341, Leu338 & Celecoxib:H28 - A:SER339:O & 2.413000 \\
\hline Celecoxih & Ser339, Gln178, Ala513 & A:ARG499:HH11 - Celecoxib:O6 & 2.308000 \\
\hline Celecoxib & Gly512, Tyr371, Val509 & Celecoxib:H28 - A:HIS75:NE2 & 2.387000 \\
\hline & His75, Trp373, Phe504 & A:PHE504:HN - Celecoxib:N9 & 2.081000 \\
\hline & Gln178 & Celecoxib:H28 - A:PHE504:HN & 1.396000 \\
\hline
\end{tabular}


Antiinflammatory activity: The compounds with good Libdock scores (Table-2) were selected for antiinflammatory activity using carrageenan-induced rat paw edema model and exhibited protection against carrageenan-induced edema. The protection ranged up to $70 \%$, while the reference drug (indomethacin) showed $76 \%$ at an equivalent dose. Among all the tested compounds, 3-(1-(2-cyanobenzyloxyimino)ethyl)coumarin showed remarkable antiinflammatory activity compared to other derivatives. This compound has cyano group at the ortho-position of aromatic ring showed enhanced activity compared to other aromatic analogues (Table-3).

TABLE-2

LibDock SCORES OF THE COMPOUNDS

\begin{tabular}{cccc}
\hline Compd. No. & $\begin{array}{c}\text { Electrostatic } \\
\text { energy }\end{array}$ & $\begin{array}{c}\text { van der } \\
\text { Waals energy }\end{array}$ & $\begin{array}{c}\text { LibDock } \\
\text { score }\end{array}$ \\
\hline $\mathbf{1 4}$ & 3.562 & 3.861 & 137.534 \\
$\mathbf{1 5}$ & -2.752 & 3.359 & 130.704 \\
$\mathbf{1 6}$ & -1.316 & 3.297 & 130.123 \\
$\mathbf{1 7}$ & 2.997 & 4.745 & 135.833 \\
$\mathbf{1 8}$ & -4.282 & 3.059 & 135.833 \\
$\mathbf{1 9}$ & -1.752 & 5.172 & 133.259 \\
$\mathbf{2 0}$ & 4.079 & 4.555 & 139.171 \\
$\mathbf{2 1}$ & -5.834 & 3.847 & 120.812 \\
$\mathbf{2 2}$ & 3.938 & 3.836 & 124.802 \\
$\mathbf{2 3}$ & 2.859 & 4.81 & 141.261 \\
$\mathbf{2 4}$ & 3.635 & 3.647 & 133.009 \\
$\mathbf{2 5}$ & -6.087 & 3.572 & 138.151 \\
Celecoxib & 4.465 & 4.762 & 145.691 \\
\hline
\end{tabular}

\begin{tabular}{ccc}
\multicolumn{3}{c}{ TABLE-3 } \\
\multicolumn{3}{c}{$\begin{array}{c}\text { ANTI-INFLAMMATORY ACTIVITY } \\
\text { OF THE TESTED COMPOUNDS* }\end{array}$} \\
\cline { 2 - 3 } Treatment & \multicolumn{2}{c}{ Volume of Paw edema $(\mathrm{mL})$} \\
\hline Control & $0.93 \pm 0.01$ & $4 \mathrm{~h}$ \\
\hline Indomethacin & $0.22 \pm 0.01(76.06)$ & $0.19 \pm 0.02(78.64)$ \\
$\mathbf{1 4}$ & $0.36 \pm 0.03(60.87)$ & $0.35 \pm 0.03(60.67)$ \\
$\mathbf{1 7}$ & $0.37 \pm 0.03(59.78)$ & $0.36 \pm 0.02(58.01)$ \\
$\mathbf{1 8}$ & $0.39 \pm 0.02(57.61)$ & $0.38 \pm 0.02(57.29)$ \\
$\mathbf{2 0}$ & $0.35 \pm 0.01(61.96)$ & $0.33 \pm 0.03(62.30)$ \\
$\mathbf{2 3}$ & $0.28 \pm 0.01(68.30)$ & $0.26 \pm 0.02(70.40)$ \\
$\mathbf{2 5}$ & $0.38 \pm 0.02(59.76)$ & $0.38 \pm 0.02(57.10)$ \\
\hline *Values are expressed as mean \pm SEM, (n $=5)$ and analyzed by \\
ANOVA. Values in parenthesis (percentage inhibition of edema).
\end{tabular}

\section{Conclusion}

Herewith, we report the simple and efficient method for synthesis of novel coumarin-oxime ether derivatives (14-25). The present methodology used to synthesize oxime ether derivatives which is the first example in coumarin chemistry can be further explored for the synthesis of various heterocyclecoumarin oxime ether derivatives. All the synthesized compounds were docked with 3LN1 and Libdock scores are measured. The compounds which got the good libDock score evaluated for their in vivo anti-inflammatory activity. The compound 3(1-(2-cyanobenzyloxyimino)ethyl)coumarin (23) exhibited good antiinflammatory activity.

\section{ACKNOWLEDGEMENTS}

The authors express their thanks to The Head, Department of University College of Technology, Osmania University and
Management of RBVRR Women's College of Pharmacy for their constant support and for providing necessary facilities.

\section{REFERENCES}

1. K.N. Venugopala, V. Rashmi and B. Odhav, BioMed Res. Int., Article ID 963248 (2013);

http://dx.doi.org/10.1155/2013/963248.

2. S. Sandhu, Y. Bansal, O. Silakari and G. Bansal, Bioorg. Med. Chem., 22, 3806 (2014); https://doi.org/10.1016/j.bmc.2014.05.032.

3. G. Cravotto, G.M. Nano, G. Palmisano and S. Tagliapietra, Tetrahedron Asymm., 12, 707 (2001); https://doi.org/10.1016/S0957-4166(01)00124-0.

4. L. Schio, F. Chatreaux and M. Klich, Tetrahedron Lett., 41, 1543 (2000); https://doi.org/10.1016/S0040-4039(99)02351-5.

5. A. Lacy and O. Kennedy, Curr. Pharm. Des., 10, 3797 (2004); https://doi.org/10.2174/1381612043382693.

6. A. Witaicenis, L.N. Seito, A. da Silveira Chagas, L.D. de Almeida, A.C. Luchini, P. Rodrigues-Orsi, S.H. Cestari and L.C. Di Stasi, Phytomedicine, 21, 240 (2014);

https://doi.org/10.1016/j.phymed.2013.09.001.

7. T.O. Olomola, R. Klein, N. Mautsa, Y. Sayed and P.T. Kaye, Bioorg. Med. Chem., 21, 1964 (2013); https://doi.org/10.1016/j.bmc.2013.01.025.

8. P. Anand, B. Singh and N. Singh, Bioorg. Med. Chem., 20, 1175 (2012); https://doi.org/10.1016/j.bmc.2011.12.042.

9. K. Venkata Sairam, B. M. Gurupadayya, R. S. Chandan, D. K. Nagesha and B. Vishwanathan, Curr. Drug Deliv., 13, 186 (2016); https://doi.org/10.2174/1567201812666150702102800.

10. M. Khoobi, A. Foroumadi, S. Emami, M. Safavi, G. Dehghan, B.H. Alizadeh, A. Ramazani, A. Shafiee, Chem. Biol. Drug Des., 78, 580 (2011); https://doi.org/10.1111/j.1747-0285.2011.01175.x.

11. J. Grover and S.M. Jachak, RSC Adv., 5, 38892 (2015); https://doi.org/10.1039/C5RA05643H.

12. M. Babazadeh-Qazijahani, H. Badali, H. Irannejad, M.H. Afsarian and S. Emami, Eur. J. Med. Chem., 76, 264 (2014); https://doi.org/10.1016/j.ejmech.2014.02.019.

13. S. Parthiban, V. Kabilan, V. Ramkumar and Y.T. Jeong, Bioorg. Med. Chem. Lett., 20, 6452 (2010); https://doi.org/10.1016/j.bmcl.2010.09.079.

14. J.-H. Chern, C.-C. Lee, C.-S. Chang, Y.-C. Lee, C.-L. Tai, Y.-T. Lin, K.-S. Shia, C.-Y. Lee and S.-R. Shih, Bioorg. Med. Chem. Lett., 14, 5051 (2004); https://doi.org/10.1016/j.bmcl.2004.07.084.

15. F. Delmas, M. Gasquet, P. Timon-David, N. Madadi, P. Vanelle, A. Vaille and J. Maldonado, Eur. J. Med. Chem., 28, 23 (1993); https://doi.org/10.1016/0223-5234(93)90075-P.

16. S.M. El-Gamal, S.M. Bayomi, S.M. El-Ashry, S.A. Said, A.A.-M. AbdelAziz and N.I. Abdel-Aziz, Eur. J. Med. Chem., 45, 1403 (2010); https://doi.org/10.1016/j.ejmech.2009.12.041.

17. M.R. Gannarapu, S.B. Vasamsetti, N. Punna, N.K. Royya, S.R. Pamulaparthy, J.B. Nanubolu, S. Kotamraju and N. Banda, Eur. J. Med. Chem., 75, 143 (2014); https://doi.org/10.1016/i.ejmech.2013.12.053.

18. A. Emami, A. Kebriaeezadeh, N. Ahangar and R. Khorasani, Bioorg. Med. Chem. Lett., 21, 655 (2011); https://doi.org/10.1016/j.bmcl.2010.12.021.

19. T. Chakravarti, T. Akhtar, B. Rai, M. Yadav, J. Akhtar Siddiqui, S.K. Dhar Dwivedi, R. Thakur, A.K. Singh, A.K. Singh, H. Kumar, K. Khan, S. Pal, S.K. Rath, J. Lal, R. Konwar, A.K. Trivedi, D. Datta, D.P. Mishra, M.M. Godbole, S. Sanyal, N. Chattopadhyay and A. Kumar, J. Med. Chem., 57, 8010 (2014); https://doi.org/10.1021/jm500873e

20. K. Park, K. Lee, S.-J. Park, B. Ahn, J.-C. Lee, H.Y. Cho and K.-I. Lee, Bioorg. Med. Chem. Lett., 15, 3307 (2005); https://doi.org/10.1016/j.bmcl.2005.03.082.

21. Y.P. Luo, Q. Gong, Q. Chen, G.F. Yang, Chin. J. Org. Chem., 28, 1561 (2008).

22. L.L. Jiang, C.N. Chen, Y.F. Zhou, Q. Chen, G.F. Yang, Chin. J. Org. Chem., 29, 1392 (2009).

23. M. Dai, X. Fu, F. He, L. Jiang, Y. Li, F. Liang, L. Liu, Y. Mi, Y.-C. Xu, G. Xun, X. Yan, Z. Yu and J.Y. Zhang, Heterocyclic Oxime Compounds, WO 2011/020861A1 (2011).

24. C.A. Winter, E.A. Risley and G.W. Nuss, Proc. Soc. Exp. Biol. Med., 111, 544 (1962). 